\title{
Features selection for Bayes classification of prolonged fatigue on rectus femoris muscle
}

\begin{abstract}
Sports training is very important to athletes in improving and maintaining their performances. It commonly involves high intensity exercise and requires longer time for fatigue to recover, compares to normal activity. During training, adequate rest is essential to allow recuperation and build body strength. Unfortunately, inadequate rest exposes the body to prolonged fatigue (PF). Hence, this condition needs to be managed accordingly to avoid chronic fatigue syndrome. Recent findings indicate that there are strong characteristics on surface EMG under PF conditions. Currently, the assessment is limited to glycogen breakdown, the existence of lactate and soreness. In this study, twenty participants were recruited to perform five days intensive training (IT) to induce more PF signs. The IT conducted was based on Bruce Protocol treadmill test. It was discovered that the IT successfully induces soreness, unexplained lethargy and performance decrement. Surface EMG were collected from rectus femoris muscle during daily pre and post treadmill tests. Four features were extracted from the surface EMG; mean frequency (Fmean), median frequency (Fmed), root mean square (RMS) and mean absolute value (MAV). The results indicated that all features during post exercise had greater value under PF condition and it was significant at $\mathrm{P}<; 0.05$. The features then were classified in accordance to Bayes. The results also showed that Fmed and MAV features offered good performance with $83.1 \%$ accuracy, $84.6 \%$ specificity and $80 \%$ of precision.
\end{abstract}

Keyword: Prolonged fatigue; Rectus femoris muscle; EMG 